\title{
EXPERIÊNCIA DA REDE BRASILEIRA DE TELEODONTOLOGIA
}

\author{
Brazilian Teledentistry Network Experience \\ Ana Estela Haddad'; Márcia Rendeiro²; Adélia Delfina da Motta Silva Correia ${ }^{3}$; Caren Bavaresco"; \\ Maria Celeste Morita5; Mary Caroline Skelton-Macedo ${ }^{6}$; Alexandra Maria Vieira Monteiro ${ }^{7}$
}

\begin{abstract}
Resumo Este artigo descreve a experiência da Rede Brasileira de Teleodontologia como parte das estratégias em eHealth. Várias instituições de todo o país participam ou vêm recebendo treinamento e suporte para a implantação de seus próprios núcleos de Telessaúde e Teleodontologia. Diversas iniciativas estão sendo tomadas de modo articulado, a fim de facilitar a criação e a consolidação de uma rede de colaborativa de trabalho em Telessaúde e Teleodontologia.
\end{abstract}

Palavras-chave: Telessaúde, Teleodontologia, Ensino a Distância, Redes Sociais

\begin{abstract}
This paper describes Brazilian experience of the National Teledentistry Network as part of the Brazilian eHealth Strategy. Many institutions from all over the country participate or are being trained and receiving support to constitute their own Telehealth and Teledentistry Centers. Multiple initiatives are held in an articulated plan, enabling capacity building and consolidation of a network for collaborative work in telehealth and teledentistry.
\end{abstract}

Keywords: Telehealth, Teledentistry, e-Learning, Social Networks

\section{Relato de Caso}

O Brasil adotou o ensino a distância aplicado à saúde (eHealth) e à educação, como política nacional, desde 2006. O movimento faz parte da ação nacional do Ministério da Saúde para desenvolvimento dos recursos da saúde humana. Diversas estratégias vêm reorientando as competências do profissional de saúde para o modelo da atenção à saúde, baseadas nos cuidados à atenção primária. A iniciativa do Ministério envolve os graduandos, os técnicos em saúde, os residentes e os pós-graduados. Tal política educacional segue três diretrizes: integração entre universidades e serviços de saúde, ampliação do conceito do processo saúde-doença, com base nos determinantes sociais de saúde e uma nova abordagem pedagógica embasada em metodologias ativas, incluindo as tecnologias da informação e comunicação (TIC) $)^{1,3}$.

O Brasil assumiu a saúde como um direito de cada um de seus agora 190 milhões de habitantes e sua Constituição Federal criou em 1988 o Sistema Único de Saúde (SUS). Um dos maiores desafios enfrentados pelos sistemas de saúde em geral é como lidar com o crescimento

\footnotetext{
1. Professora Adjunta na Faculdade de Odontologia da Universidade de São Paulo, Coordenadora do Programa Nacional de Telessaúde do Ministério da Saúde (2006-2012); 2. Doutora, Professora da Universidade do Estado do Rio de Janeiro, Brasil; 3. Coordenadora do Programa Estadual de Telessaúde da Secretaria de Saúde do Mato Grosso do Sul, Brasil; 4. PhD e Dentista Reguladora do Programa Estadual de Telessaúde da Universidade Federal do Rio Grande do Sul; 5. Pós-Doutora; Professora Adjunta da Universidade Estadual de Londrina - PR, presidente da Associação Brasileira de Ensino Odontológico; 6. PhD, Professora de Teleodontologia da Faculdade de Odontologia da Universidade de São Paulo, Brasil; 7. Professora Associada da Faculdade de Ciências Médicas; Coordenadora do Laboratório de Telessaúde - Universidade do Estado do Rio de Janeiro
} 
avassalador de novas tecnologias e, ao mesmo tempo, garantir o acesso universal da população aos cuidados de alta qualidade. Recursos humanos em saúde desempenham um papel central nesta equação².

As múltiplas estratégias para o eHealth incluem principalmente o Programa Telessaúde Brasil Redes (www.telessaudebrasil.org.br), a Universidade Aberta do Sistema Único de Saúde - UNA-SUS (www.unasus. gov.br) e a Rede Universitária de Telemedicina - RUTE (www.rute.rnp.br).

A Odontologia ganhou força no SUS em 2000, quando os dentistas começaram a integrar Equipes de Saúde da Família (ESF) ao nível dos cuidados de saúde primários e também com a criação dos Centros de Especialidades Odontológicas, em 2005. Em 2012, chegamos a cerca de 18 mil de um total de $32 \mathrm{mil}$ ESFs, que incluíram os dentistas e 800 Centros de Especialidades Odontológicas em todo o país. Antes disso, a maioria da população tinha dificuldades de acesso ao tratamento odontológico. Estudos epidemiológicos mostraram uma melhoria considerável nos indicadores de saúde bucal entre 2000 e 2010, embora ainda tenhamos uma grande demanda acumulada.

A inclusão da odontologia no SUS criou a necessidade de cursos de graduação para preparar melhor os estudantes para um novo padrão de atividade profissional, capacitando-os a enfrentar o desafio de trabalhar com equipes multiprofissionais e com serviços públicos e não apenas em consultórios particulares.

Um desses programas é a estratégia de eHealth. Para melhor integrar a Odontologia ${ }^{5,7}$, foi criada, em 2011, a Rede Nacional de Teleodontologia - a RNTO (http://programa.telessaudebrasil.org.br/vhl/teleodontologia-em-foco/rede-nacional-de-teleodontologiae-nucleos), cujo objetivo é compartilhar experiências bem-sucedidas de Telessaúde aplicadas a Teleodontologia, em nível nacional e no exterior. Duas iniciativas relevantes da RNTO estão incluídas. Uma delas é o curso de e-learning para o desenvolvimento de Centros de Teleodontologia. Está dividida em três módulos: 1. Como implantar e gerir um Núcleo de Teleodontologia, 2. Como treinar e estimular os professores a utilizar mais amplamente tecnologias de informação e comunicação (TIC) no processo de ensino-aprendizagem; 3. Como oferecer teleconsultorias e serviços de Segunda Opinião Formativa para profissionais de saúde. Cada módulo está estruturado estabelecendo as diretrizes e as lições aprendidas. Após a definição do conteúdo e sua validação por um comitê externo experiente, o módulo é desenvolvido com o apoio de um designer instrucional. Espera-se que este curso possa servir como referência para professores, profissionais de saúde, faculdades de Odontologia e serviços de saúde que queiram utilizar as TIC no ensino-aprendizagem e no apoio à saúde.

A segunda iniciativa, como parte da RUTE, é a criação de um Special Interest Group (SIG) em Teleodontologia. Ele representa um dos mais de 40 SIGs em atividade de diferentes especialidades, a maioria em Medicina e Enfermagem. O SIG Teleodontologia é um espaço virtual em que as instituições de ensino e pesquisa de todo o país se "reúnem" através de vídeo e webconferências para discutir e compartilhar experiências e aprendizados. Esse grande movimento de educação e pesquisa em saúde foi possível graças a uma iniciativa de cooperação executada pelo Ministério da Ciência e Tecnologia, juntamente com os Ministérios da Educação e da Saúde. Um sistema integrado de banda larga foi constituído, ligando todos os hospitais públicos universitários e outros serviços de saúde relevantes: a Rede Nacional de Ensino e Pesquisa (www.rnp.br), responsável pelo desenvolvimento e gestão da RUTE.

A RNTO é conduzida pela Associação Brasileira de Ensino Odontológico (www.abeno.org.br) e pelo Núcleo de Teleodontologia da Faculdade de Odontologia da Universidade de São Paulo (www.teleodonto.fo.usp.br) Teleodontologia, em parceria com os núcleos de 
Telessaúde da Universidade do Estado do Rio de Janeiro (http://www.telessaude.uerj.br), da Universidade Federal do Rio Grande do Sul (http://www.ufrgs.br/telessauders) e com a Secretaria de Saúde de Mato Grosso do Sul (http://telessaude.saude.ms.gov.br). O projeto é patrocinado pelo Ministério da Saúde (www.saude.gov.br), com a colaboração da Organização Pan-Americana de Saúde. Ambas as iniciativas são articuladas para o fortalecimento da RNTO, contribuindo também para a melhoria dos cuidados em saúde, para a pesquisa e a educação na graduação, bem como nos cursos de pósgraduação em Odontologia em todo o país.

\section{Referências}

1. A.E. Haddad, F.E. Campos, G.C. Cury, J.R. Ferreira. “Pró-Saúde - Programa nacional de reorientação da formação profissional em saúde: objetivos, implementação e desenvolvimento potencial.." Brasília, DF: Ministério da Saúde; 2007. 87 p.
2. A.E. Haddad "A Odontologia na Política de Formação dos Profissionais de Saúde, o papel da Teleodontologia como ferramenta do processo de ensino-aprendizagem e a criação do Núcleo de Teleodontologia da FOUSP." Tese de Livre-Docência. Faculdade de Odontologia da USP. 2011. 198p.

3. N. Almeida-Filho. “Higher education and health care in Brazil." Lancet v. 377(9781), p.1898-900. June 2011.

4. A.E. Haddad "Brazilian Experience in Telehealth Brazil Program." In: Gold Book 50 Congresso Científico do HUPE. eBook. Tecnologia em Educação e Saúde. 2012. http://www.telessaude.uerj.br/goldbook/artigos/

5. M. Bradley, P. Black, R. Noble, R. Thompson, P. J. Lamey. “Application of Teledentistry in oral medicine in a Community Dental Service, Northern Ireland". Br Dent J. vol. 209(8), p.399-404 2010.

6. A.E. Haddad, M.C. Skelton-Macedo, C.A. Costa, P.E. Souza, L.W. Chao, F.E. Campos. "Brazil Telehealth Program in the Amazon Region: Brazilian Strategy to Reach isolated populations." Global Telemed eHealth Updates: knowledge Resources vol.3 p.64-8. 2010.

7. A.E. Haddad, A.C. Guedes-Pinto, C.J.F. Alencar, E. Sequeira, J.H. Antoniazzi, L.B. Oliveira, et al. Teleodontologia: potencialidades no ensino e na prática odontológica. In: Guedes-Pinto AC, Bonecker M, Rodrigues CRMD (Org.). Fundamentos da odontologia: odontopediatria. 1. ed. São Paulo: Santos; 2009. v. 1, p. 437-45.

8. A.E. Haddad, M.C. Skelton-Macedo, E.D. Castro Filho, V. Abdala, R.M.P. Andrade, F.E. Campos. "Formative Second Opinion: Qualifying Primary Health Care in Brazil." Global Telemed eHealth Updates: knowledge Resources. 2010;3:546-50. 